\title{
Estudio termodinámico en la obtención de nanohidroxiapatita por el método de microondas $\diamond$
}

\author{
L. S. Villaseñór Cerón ${ }^{a+}$, D. Mendoza-Anaya ${ }^{b}$, M.I. Reyes-Valderrama ${ }^{a}$, E. Salinas-Rodríguez ${ }^{\mathrm{a}}$, V. Rodríguez-Lugo ${ }^{\text {a* }}$ \\ a Área Académica de Ciencias de la Tierra y Materiales, Universidad Autónoma del Estado de Hidalgo, Carr. Pachuca-Tulancingo km 4.5, C.P. 42184 Pachuca, \\ Hidalgo, México. \\ ${ }^{b}$ Instituto Nacional de Investigaciones Nucleares, Carr. México-Toluca S/N, La Marquesa, Ocoyoacac, Edo. de México C.P. 52750, México.
}

\begin{abstract}
Resumen
En el presente trabajo se lleva a cabo la síntesis de nanohidroxiapatita a través del método hidrotermal asistido por microondas, utilizando como precursores: nitrato de calcio tetrahidrato $\left(\mathrm{Ca}\left(\mathrm{NO}_{3}\right)_{2} \cdot 4 \mathrm{H}_{2} \mathrm{O}\right)$, fosfato de di-hidrogeno amonio $\left(\left(\mathrm{NH}_{4}\right) \mathrm{H}_{2} \mathrm{PO}_{4}\right)$ e hidróxido de amonio $\left(\mathrm{NH}_{4} \mathrm{OH}\right)$, utilizando un $\mathrm{pH}$ de 11 a una temperatura de $150{ }^{\circ} \mathrm{C}$, que se establecieron a partir de cálculos termodinámicos en relación con la energía libre de Gibbs, variando el tiempo de reacción de 15, 30 y 60 minutos. Los materiales obtenidos se caracterizaron por las siguientes técnicas: Difracción de Rayos X (DRX) obteniendo los planos cristalinos (002), (300) y (202) característicos de la hidroxiapatita con estructura hexagonal, de acuerdo la ficha JPDF 82-1943 y finalmente caracterizado por Microscopía Electrónica de Barrido (MEB) que mostró estructuras con forma aglomerados con tamaños que oscilan entre 4 y $0,3 \mu \mathrm{m}$. Por lo tanto, a partir de la energía libre de Gibbs calculada, $\Delta \mathrm{G}=-7222763 \mathrm{~J} / \mathrm{mol}$, se obtienen las condiciones óptimas de trabajo para generar las nanoestructuras deseadas.
\end{abstract}

Palabras Clave: Hidroxiapatita, Microondas y Termodinámica

\section{Introducción}

En la actualidad los nanomateriales han tenido un gran interés científico, debido a las características y propiedades que estos exhiben al encontrase dentro del rango nanométrico $\left(1 \times 10^{-9} \mathrm{~m}\right.$.), este tipo de materiales han llegado a presentar mejores propiedades ópticas [1], eléctricas, mecánicas y catalíticas [2], entre otras. Por lo que diversas investigaciones se han centrado en la síntesis de nanoestructuras que les permitan modificar sus propiedades iniciales, con la finalidad de mejorar la salud humana, $y$, por lo tanto, la calidad de vida.

Tal es el caso de la hidroxiapatita (HAp), un biomaterial, que presenta propiedades tales como; Termoluminiscencia [3], biocompatibilidad [4], osteointegración [5], etc., las cuales están relacionadas directamente con el tamaño y la morfología del nanomaterial. El control del tamaño y forma de partícula depende del método y la optimización de las diferentes variables implicadas en la síntesis de HAp, que contribuyen a mejorar las propiedades de biocompatibilidad, favoreciendo a que el nanomaterial presente características para aplicaciones como biomaterial en tratamientos médicos alternativos [6], injertos [7] e implantes [8] evitando el rechazo del organismo al material.

El campo de aplicación de HAp depende directamente de las propiedades, las cuales están relacionadas intrínsicamente con las características microestructurales y fisicoquímicas de la HAp, y a su vez están vinculadas con el método de síntesis; es común observar morfologías tales como: esferas, agujas, fibras, entre otros [9]. Existen otros factores que de igual forma intervienen en la morfología y el crecimiento de HAp, y son los parámetros de síntesis: concentración de los reactivos, $\mathrm{pH}$, tiempo y temperatura de reacción [10,11], así como las posibles fuentes de calcio y fosforo (precursores) [12,13]. Los métodos de síntesis HAp pueden clasificarse de la siguiente manera: métodos secos, donde no se utilizan disolventes y los productos obtenidos son polvos con tamaños superiores a la escala nanométrica y forma de partícula irregular; generalmente estas características no están directamente relacionadas con los parámetros del proceso ya que no requiere un preciso control de éste y tampoco se requieren altas presiones [14]. Por otro lado, los métodos húmedos han sido ampliamente utilizados para sintetizar HAp nanoestructurada, con forma y tamaño de partícula controlado. Se les conoce como un proceso simple en el que el comportamiento y el crecimiento del material están directamente relacionados con los parámetros de reacción [15]. Métodos de alta temperatura, que son fácilmente identificables debido a que utilizan temperaturas que lleven a la descomposición química de los componentes, es decir, a la pirolisis, donde se requiere un control estricto en los parámetros del proceso; se obtienen polvos de HAp altamente cristalinos [16]. Dentro de los procesos húmedos se pueden encontrar el método hidrotermal asistido por microondas, que constituye una técnica alternativa que aporta energía térmica a una reacción con ventajas como el ahorro de tiempo y energía. Este método consiste en someter la muestra a altas temperaturas y presiones en presencia de radiación de microondas, resultando en la generación de menos subproductos, es decir, optimizando 
los rendimientos, en un menor número de pasos; actualmente se ha constituido como un método alternativo que apoya el desarrollo de nuevas líneas de investigación ya que permite la optimización de las condiciones de síntesis [17,18]. Por ejemplo, de acuerdo a trabajos previos se ha concluido que la temperatura óptima para la formación de nanobarras de HAp es a $200^{\circ} \mathrm{C}$ a un $\mathrm{pH} 10$ por $30 \mathrm{~min}$ de reacción $[19,20]$. Uno de los inconvenientes de este método es que la temperatura requerida es lo suficientemente alta produciendo daños en los reactores de síntesis, lo cual se busca disminuir la temperatura sin alterar la energía de formación.

Diversas investigaciones se han enfocado en el estudio de las propiedades termodinámicas del material para establecer la energía necesaria a la cual se lleve a cabo la reacción [21-23], Cualquier cambio en las condiciones del sistema de síntesis, conlleva a un cambio en las energías de reacción, por lo consiguiente si se desea implementar la síntesis de HAp a menores temperaturas, se debe evaluar la energía que se requiere para la formación de la HAp a las condiciones óptimas $\left(200^{\circ} \mathrm{C}\right.$ por $30 \mathrm{~min}$.) y buscar un ajuste en la energía a menores temperaturas, variando factores como el $\mathrm{pH}$ y tiempo de reacción, lo cual es posible a través de la energía libre de Gibbs.

En el presente trabajo, se estudió la síntesis de HAp por el método hidrotermal asistido por microondas, a partir de los cálculos de energía libre de Gibbs, variando el pH y el tiempo de reacción. El propósito de esta investigación es establecer un control eficiente en términos de tamaño y morfología de las nanoestructuras. Los resultados obtenidos en este trabajo podrían servir como base para la obtención de nanoestructuras en condiciones óptimas a partir del cálculo de la energía libre de Gibbs.

\section{Metodología}

\subsection{Cálculos Termodinámicos}

Se realizó un análisis termodinámico utilizando la energía libre de Gibbs como base para lograr las condiciones óptimas para la obtención de HAp de acuerdo con la reacción establecida en la ecuación 1. Posteriormente se realizó el cálculo correspondiente a la energía libre de Gibbs, expresada en la ecuación 2 en donde se estableció que $\Delta \mathrm{G}$ a $298 \mathrm{~K}$ (25 ${ }^{\circ} \mathrm{C}$ ) de la hidroxiapatita es $-13709000 \mathrm{~J} / \mathrm{mol}$, para $\mathrm{Ca}^{+2}$ es $526830 \mathrm{~J} / \mathrm{mol}$, para $\mathrm{PO}_{4}{ }^{-3}$ es a $-1211700 \mathrm{~J} / \mathrm{mol}$, y para el $\mathrm{H}_{2} \mathrm{O}$ es de $-306110 \mathrm{~J} / \mathrm{mol}$; dichos datos fueron obtenidos de la base de datos del software SAT-IPN.exe.

$$
\begin{gathered}
\mathrm{H}_{2} \mathrm{O}+3 \mathrm{PO}_{4}^{-3}+5 \mathrm{Ca}^{+2} \rightarrow \mathrm{Ca}_{5}\left(\mathrm{PO}_{4}\right)_{3}(\mathrm{OH})+\mathrm{H}^{+} \\
\Delta G^{\circ}=\Delta G_{\text {productos }}-\Delta G_{\text {reactivos }}
\end{gathered}
$$

El resultado obtenido en la ecuación 2 fue sustituido en la ecuación 3 , a una temperatura de $373 \mathrm{~K}\left(100{ }^{\circ} \mathrm{C}\right)$, 423K $(150$ $\left.{ }^{\circ} \mathrm{C}\right)$ y $473 \mathrm{~K}\left(200{ }^{\circ} \mathrm{C}\right)$, variando el $\mathrm{pH}=10,11$ y 12 .

$$
\Delta G=\Delta G^{\circ}+R T \ln (K)
$$

Posteriormente se compararon las energías obtenidas a diferentes temperaturas y $\mathrm{pH}$ como se muestra en la tabla 1.

\begin{tabular}{|c|c|c|c|}
\hline & & Temperatura $\left({ }^{\circ} \mathrm{C}\right)$ & $\Delta \mathrm{G}(\mathrm{J} / \mathrm{mol})$ \\
\hline \multirow{9}{*}{$\mathrm{pH}$} & 10 & \multirow{3}{*}{100} & 7205087.5 \\
\hline & 11 & & -7212232.3 \\
\hline & 12 & & -7219377.0 \\
\hline & 10 & \multirow{3}{*}{150} & -7214661.1 \\
\hline & 11 & & -7222763.2 \\
\hline & 12 & & -7230865.3 \\
\hline & 10 & \multirow{3}{*}{200} & -7224234.7 \\
\hline & 11 & & -723329.1 \\
\hline & 12 & & -7242353.6 \\
\hline
\end{tabular}

Tabla 1: A partir de los datos obtenidos del cálculo de $\Delta \mathrm{G}$, variando temperatura y $\mathrm{pH}$.

En la Tabla 1 se visualizan los resultados obtenidos del análisis termodinámico, en el cual se obtiene que a una temperatura de $150{ }^{\circ} \mathrm{C}$ a un $\mathrm{pH} 11$ es posible la obtención de nanobarras de HAp, debido a que es la energía que más se acerca a $\mathrm{T}=200{ }^{\circ} \mathrm{C}$ a $\mathrm{pH}=10$ (condiciones óptimas), anteriormente descritas.

Por lo consiguiente se varían los tiempos de reacción a 15,30 y $60 \mathrm{~min}$, debido a la pequeña variación entre energías.

\subsection{Síntesis de Hidroxiapatita}

La síntesis de HAp se llevó a cabo a partir de los precursores de nitrato de calcio tetrahidratado $\left(\mathrm{Ca}\left(\mathrm{NO}_{3}\right)_{2} 4 \mathrm{H}_{2} \mathrm{O}\right)$ (Meyer-99.0\%), fosfato di-hidrógeno de amonio $\left(\left(\mathrm{NH}_{4}\right) \mathrm{H}_{2} \mathrm{PO}_{4}\right)$ (JT Baker-98.0\%) e hidróxido de amonio $\left(\mathrm{NH}_{4} \mathrm{OH}\right)$ (JT Baker-28.0-30, 0\%), a través del método hidrotermal asistido por microondas. Las soluciones se prepararon con agitación constante (Figura1-a). Se añadió Ca $\left(\mathrm{NO}_{3}\right)_{2} 4 \mathrm{H}_{2} \mathrm{O}$ a la solución a una concentración de $0.10 \mathrm{M}$ (Figura 1-b) dispersada en $53.4 \mathrm{ml}$ de agua destilada, luego se añadió $0.10 \mathrm{M}$ de $\left(\mathrm{NH}_{4}\right) \mathrm{H}_{2} \mathrm{PO}_{4}$ a la solución (Figura1-c). Posteriormente el hidróxido de amonio fue agregado gota por gota (Figura1-d), para aumentar el pH a 11. La solución obtenida es colocada en un reactor de Teflón, el cual que se introducirá al microondas; la síntesis se llevará a cabo a una temperatura de $150^{\circ} \mathrm{C}$, con tiempos de 15, 30 y $60 \mathrm{~min}$ (HAP-15, HAP-30 y HAP-60) (Fig. 1-e). Finalmente, las muestras son lavadas y secadas (Figura1-f). 


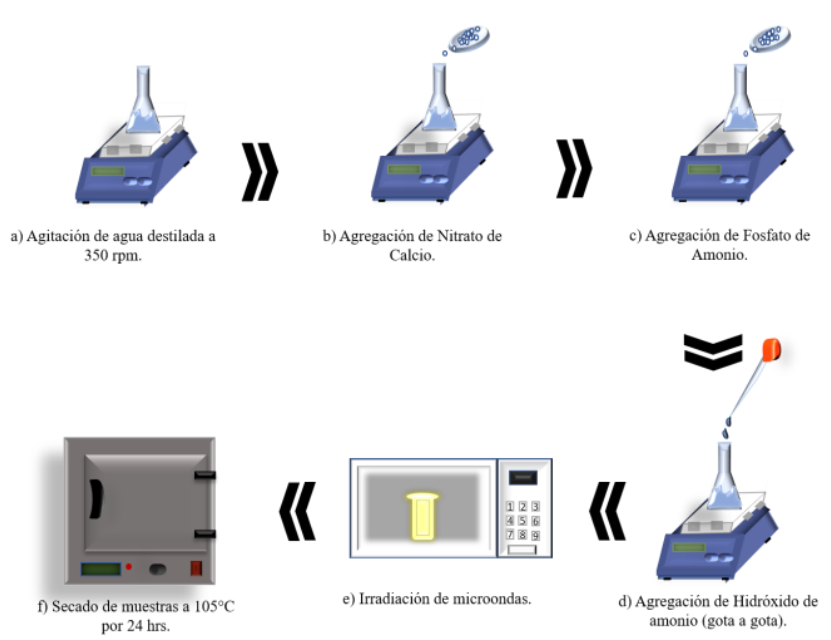

Figura 1: Síntesis de HAp a) agitación constante de agua a 350 RPM, b) adición de nitrato de calcio, c) adición de fosfato de amonio, d) gota por gota de hidróxido de amonio e) la síntesis del HAp a una temperatura de $150^{\circ} \mathrm{C}$, con un tiempo de 15,30 y 60 mín. y f), la muestra se lava y seca.

\subsection{Caracterización}

Los productos sintetizados, fueron caracterizados por las técnicas de Difracción de Rayos X (DRX), utilizando un Difractómetro marca Bruker, modelo D8 Discover, el cual se utilizó para obtener las fases cristalinas presentes y el tamaño cristal. Por Microscopia Electrónica de Barrido (MEB) se observó la morfología de la HAp utilizando un Microscopio marca Jeol, Modelo JSM7800FE, en modo de detección de electrones secundarios. Adicionalmente, el software "Image J" se utilizó para calcular el tamaño de partícula promedio de la HAp [24].

\section{Resultados}

Las muestras de HAp (HAp-15, HAp-30 y HAp-60) se sintetizaron por el método hidrotermal asistido por microondas a diferente tiempo de reacción, para observar su comportamiento estructural y morfológico. Se caracterizaron por DRX para determinar la estructura y fases cristalinas de los productos obtenidos, y por MEB se determinó el tamaño de partícula y la morfología del material.

\subsection{Difracción de Rayos X.}

El patrón de difracción de rayos $\mathrm{X}$ de las muestras sintetizadas a diferentes tiempos de reacción se muestra en la figura 2, en la que se observan los difractogramas correspondientes, donde se identifica la fase hexagonal de acuerdo a la tarjeta JPDF 82-1943 y se distinguen tres planos cristalográficos principales de la HAp, presentes en $28,8^{\circ}$, $31,6^{\circ}$ y $32,7^{\circ}$, en $2 \theta$, correspondiente a los planos (210), (211) y (300) respectivamente. De igual forma se aprecian planos cristalinos presentes a $18,06^{\circ}, 28,6^{\circ}, 34,1^{\circ}, 36,6^{\circ}, 50,7^{\circ}$ y $64,3^{\circ}$ en $2 \theta$, correspondientes a la fase hexagonal del hidróxido de calcio, que forma parte de los precursores utilizados durante la síntesis.

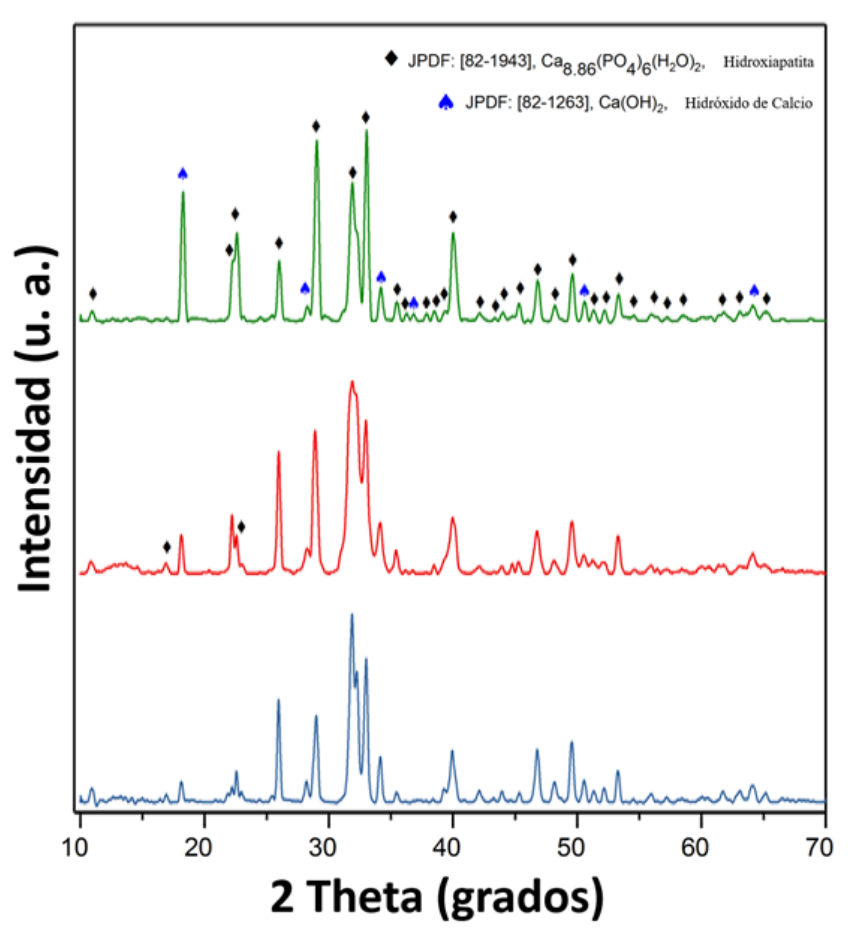

Figura 2: Difractograma de la síntesis de HAp a diferentes tiempos de reacción; 15, 30 y $60 \mathrm{~min}$.

El incremento del tiempo de reacción disminuye la intensidad de los planos identificados del hidróxido de calcio e incrementan la intensidad de los planos cristalográficos de la HAp, lo que indica que, para un tiempo de reacción de 60 min, se favorece la obtención de HAp con mayor cristalinidad y con menos presencia de productos secundarios.

El promedio del tamaño de cristal se calculó según la ecuación de Debye-Scherrer (ecuación 3), utilizando los principales planos cristalográficos; (210), (211) y (300) de las tres muestras sintetizadas, mostradas en la tabla2.

$$
D=\frac{x \cdot \lambda}{B \cdot \operatorname{Cos} \theta}
$$

Tabla 2: Tamaño de cristal.

\begin{tabular}{cc} 
Muestra & $\begin{array}{c}\text { Tamaño de cristal } \\
\text { (nm) }\end{array}$ \\
\hline \hline HAp-15 & 7.13 \\
HAp-30 & 9.63 \\
HAp-60 & 14.26
\end{tabular}

Se observa un incremento del tamaño del cristal el cual se relaciona con el incremento del tiempo de reacción. Para la muestra de HAp-15 se obtuvo un tamaño de cristal de 7.13nm, para la muestra HAp-30 se determinó un tamaño de cristal de $9.63 \mathrm{~nm}$ y finalmente el tamaño de cristal de la muestra HAp60 es de $14.26 \mathrm{~nm}$.

\subsection{Microscopia Electrónica de Barrido}

Las muestras HAP-15, HAP-30 y HAP-60 se caracterizaron por microscopía electrónica de barrido, las 
cuales se muestran en la figura 3 , donde se observa un conjunto de aglomerados. En la Figura 3a-c, el tamaño de partícula HAp disminuye con el aumento del tiempo de reacción. El tamaño de partícula promedio para HAP-15 es alrededor de $300 \mathrm{~nm}$ y para las muestras HAP-30 y HAP-60 es de aproximadamente 3 a $4 \mu \mathrm{m}$ respectivamente. El tamaño promedio de partículas se obtuvo con el software Image $\mathbf{J}$.

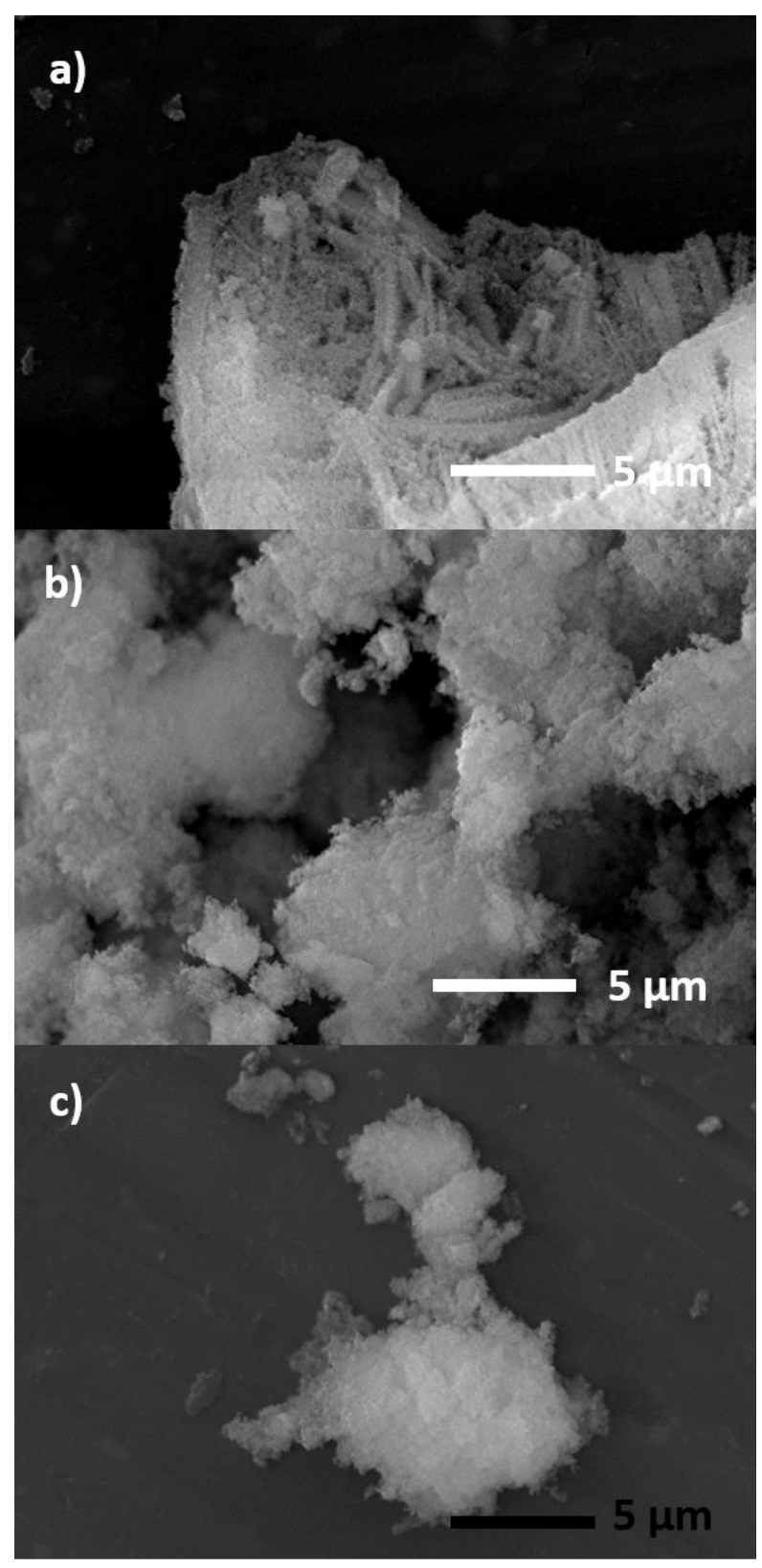

Figura 3: Micrografías obtenidas por microscopía electrónica de barrido de las muestras (a) HAP-15, (b) HAP-30 y (c) HAP-60.

Los tamaños de partícula se incrementan con el aumento del tiempo de reacción. En la figura 3a, se visualizan estructuras aún más pequeñas, donde no es posible apreciar detalles de éstas debido a la resolución del equipo. Es decir, no fue posible determinar la microestructura que conforman los aglomerados. Por lo que en una segunda etapa de investigación se propone incorporara técnica como microscopia electrónica de transmisión de alta resolución.

\section{Conclusiones}

La energía libre de Gibbs, que según los cálculos en este estudio fue de $\Delta \mathrm{G}-7224234.7 \mathrm{~J} / \mathrm{mol}$, permite determinar las condiciones de equilibrio y de espontaneidad para una reacción química minimizando las pruebas de laboratorio para la obtención de HAp. Es decir, el cálculo de la energía libre de Gibbs es una herramienta importante para realizar el estudio termodinámico, encontrando las condiciones óptimas de síntesis. La variación de los parámetros como el tiempo y el $\mathrm{pH}$, son factores básicos que favorecen el crecimiento de HAp, para tiempos de reacción de 15, 30 y 60 minutos por método de microondas donde se aprecia el incremento de los aglomerados de 0,3 a $4 \mu \mathrm{m}$. Y de acuerdo a los resultados obtenidos por DRX, es posible establecer que se llevó a cabo la síntesis de HAp, sin productos secundarios, a una temperatura de $150{ }^{\circ} \mathrm{C} \mathrm{y} \mathrm{a} \mathrm{pH}=11$, lo que muestra que si fue viable la obtención de HAp en estas condiciones.

\section{English Summary}

Thermodynamic study for the obtention of Nanohydroxyapatite by the microwave method.

\begin{abstract}
In the present work focuses on the analysis and synthesis of nanohydroxyapatite using the microwave-assisted hydrothermal method, and calcium nitrate tetrahydrate $\left(\mathrm{Ca}\left(\mathrm{NO}_{3}\right)_{2} \cdot 4 \mathrm{H}_{2} \mathrm{O}\right)$, diammonium phosphate $\left(\left(\mathrm{NH}_{4}\right)_{2} \mathrm{HPO}_{4}\right)$ and ammonium hydroxide $\left(\mathrm{NH}_{4} \mathrm{OH}\right)$ as precursors, with a $\mathrm{pH}$ of 11.2 and a temperature of $150{ }^{\circ} \mathrm{C}$, which were previously established from thermodynamic calculations in relation to Gibbs free energy; the reaction time was 15,30 and 60 minutes. The materials obtained were characterized by X-ray diffraction (XRD) and scanning electron microscopy (SEM) techniques. XRD showing presence of crystalline planes at (002), (300) and (202) associates with the hexagonal hydroxyapatite phase, which was indexing with the JPDF 82-1943 card. On the other hand, SEM showed agglomerates with irregular morphologies and sizes ranging between 4 and $0.3 \mu \mathrm{m}$. Therefore, from the calculated Gibbs free energy, $\Delta \mathrm{G}=-7222763$, optimal working conditions are obtained to generate the desired nanostructures.
\end{abstract}

Keywords:

Hydroxyapatite, microwaves and thermodynamics.

\section{Agradecimientos}

Los autores agradecen al Instituto Nacional de investigación nuclear (ININ) por el uso de sus instalaciones de laboratorio y caracterización por MEB y DRX. Así como al Consejo Nacional de ciencia y tecnología (CONACYT) por el soporte económico e intelectual a través del programa de Doctorado en Ciencia de los Materiales en el área académica de Ciencias de 
la Tierra y Materiales de la Universidad Autónoma del Estado de Hidalgo

\section{Referencias}

[1] J.Z. Zhang, Optical Properties and Spectroscopy of Nanomaterials, WORLD SCIENTIFIC, 2009. doi:10.1142/7093.

[2] M.L. Doroteo, Nanomateriales: Nuevas propiedades a menores dimensiones, (2003). http://www.revistaciencia.amc.edu.mx/images/revis ta/54_1/nanomateriales.pdf (accessed July 4, 2018).

[3] M.A. Vallim De Alencar, The TL and OSL study of hydroxyapatite for dosimetric applications, (2009).

http://www.iaea.org/inis/collection/NCLCollection Store/_Public/41/113/41113433.pdf (accessed July 19, 2017).

[4] Mehdi Sadat-Shojai, Mohammad-Taghi Khorasani, Ehsan Dinpanah-, Khoshdargi, Ahmad Jamshidi, Synthesis methods for nanosized hydroxyapatite with diverse structures, Acta Biomater. 9 (2013) 7591-7621. doi:10.1016/J.ACTBIO.2013.04.012.

[5] K. Breding, R. Jimbo, M. Hayashi, Y. Xue, K. Mustafa, M. Andersson, The effect of hydroxyapatite nanocrystals on osseointegration of titanium implants: an in vivo rabbit study., Int. J. Dent. 2014 (2014) 171305.

doi:10.1155/2014/171305.

[6] K. Okuda, H. Tai, K. Tanabe, H. Suzuki, T. Sato, T. Kawase, Y. Saito, L.F. Wolff, H. Yoshiex, Platelet-Rich Plasma Combined With a Porous Hydroxyapatite Graft for the Treatment of Intrabony Periodontal Defects in Humans: A Comparative Controlled Clinical Study, J. Periodontol. 76 (2005) 890-898. doi:10.1902/jop.2005.76.6.890.

[7] P. Oshino, Tomihisa MD, PHD; Murase, Tomoo MD, PHD; Saito, Tomoyuki MD, Medical opening-wedge high tibial osteotomy with use of porous hydroxyapatite to treat medial compartment osteoarthritis of the knee, J. Bone Jt. Surg. 85 (2003) 78-85.

https://journals.lww.com/jbjsjournal/subjects/Foot and

Ankle/Abstract/2003/01000/MEDIAL_OPENING_ WEDGE_HIGH_TIBIAL_OSTEOTOMY_WITH. 13.aspx (accessed June 8, 2018).

[8] L. Xia, Y. Xie, B. Fang, X. Wang, K. Lin, In situ modulation of crystallinity and nano-structures to enhance the stability and osseointegration of hydroxyapatite coatings on Ti-6Al-4V implants, Chem. Eng. J. 347 (2018) 711-720. doi:10.1016/J.CEJ.2018.04.045.

[9] X. Zhang, K.S. Vecchio, Hydrothermal synthesis of hydroxyapatite rods, J. Cryst. Growth. 308 (2007) 133-140. doi:10.1016/j.jcrysgro.2007.07.059.

[10] V. Rodríguez-Lugo, T.V.K. Karthik, D. Mendoza-
Anaya, E. Rubio-Rosas, L.S. Villaseñor Cerón, M.I. Reyes-Valderrama, E. Salinas-Rodríguez, Wet chemical synthesis of nanocrystalline hydroxyapatite flakes: Effect of $\mathrm{pH}$ and sintering temperature on structural and morphological properties, R. Soc. Open Sci. 5 (2018). doi:10.1098/rsos.180962.

[11] A. Sáenz, M.L. Montero, G. Mondragón, V. Rodríguez-Lugo, V.M. Castaño, Effect of pH on the precipitation of hydroxyapatite on silica gels, Mater. Res. Innov. 7 (2003) 68-73. doi:10.1007/s10019-003-0230-x.

[12] V. Rodríguez-Lugo, J. Sanchez Hernández, M.J. Arellano-Jimenez, P.H. Hernández-Tejeda, S. Recillas-Gispert1, Microscopy Microanalysis Characterization of Hydroxyapatite by Electron Microscopy, Scanning. 11 (2005) 516-523. doi:10.1017/S1431927605050646.

[13] V. Rodríguez-Lugo, C. Angeles-Chavez, M. Hernandez, Synthesis of hydroxylapatite from sand dollar and $\beta$-tricalcium phosphate by solid-state method, Mater. Manuf. Process. 18 (2003) 903913. doi:10.1081/AMP-120025078.

[14] S. Pramanik, A.K. Agarwal, K.N. Rai, A. Garg, Development of high strength hydroxyapatite by solid-state-sintering process, (2006). doi:10.1016/j.ceramint.2005.10.025.

[15] T. İnce, O. Kaygili, C. Tatar, N. Bulut, S. Koytepe, T. Ates, The effects of Ni-addition on the crystal structure, thermal properties and morphology of Mg-based hydroxyapatites synthesized by a wet chemical method, Ceram. Int. (2018). doi:10.1016/J.CERAMINT.2018.04.257.

[16] J.S. Cho, S.-H. Rhee, Formation mechanism of nano-sized hydroxyapatite powders through spray pyrolysis of a calcium phosphate solution containing polyethylene glycol, J. Eur. Ceram. Soc. 33 (2013) 233-241.

doi:10.1016/J.JEURCERAMSOC.2012.08.029.

[17] M. de las nieves Zavala, Síntesis asistida por Microondas, Cent. Conjunto Investig. En Química Sustentable. (2008).

http://www.cciqs.unam.mx/index.php?option=com _content \&view $=$ article \&id=85\&Itemi $\mathrm{d}=87$ (accessed September 7, 2017).

[18] J. Prado-Gonjal, Microwave - assisted synthesis and characterization of inorganic materials, (2014). doi:10.13140/2.1.1646.1766.

[19] V. Rodríguez-Lugo, E. Salinas-Rodríguez, R.A. Vázquez, K. Alemán, A.L. Rivera, Hydroxyapatite synthesis from a starfish and $\beta$-tricalcium phosphate using a hydrothermal method, RSC Adv. 7 (2017) 7631-7639. doi:10.1039/C6RA26907A.

[20] A. Shavandi, A.E.-D.A. Bekhit, A. Ali, Z. Sun, Synthesis of nano-hydroxyapatite (nHA) from waste mussel shells using a rapid microwave method, Mater. Chem. Phys. 149-150 (2015) 607616.

doi:10.1016/J.MATCHEMPHYS.2014.11.016. 
[21] M.G.J. Ten Cate, J. Huskens, M. Crego-Calama, D.N. Reinhoudt, Thermodynamic stability of hydrogen-bonded nanostructures: A calorimetric study, Chem. - A Eur. J. 10 (2004) 3632-3639. doi:10.1002/chem.200400085.

[22] H.R. Mahmoud, S.M. Ibrahim, S.A. El-Molla, Textile dye removal from aqueous solutions using cheap $\mathrm{MgO}$ nanomaterials: Adsorption kinetics, isotherm studies and thermodynamics, Adv. Powder Technol. 27 (2016) 223-231. doi:10.1016/j.apt.2015.12.006.

[23] E. Lerner, S. Sarig, Enhanced maturation of hydroxyapatite from aqueous solutions using microwave irradiation, 1991.

https://link.springer.com/content/pdf/10.1007\%2FB F00692971.pdf (accessed May 11, 2019).

[24] J.-Y. Ruzicka, Particle sizing using ImageJ MESA, (2013). http://mesa.ac.nz/2013/03/particlesizing-using-imagej/ (accessed August 2, 2017). 